\title{
EFFECT OF MYCORRHIZAL INOCULATION AND SUBSTRATE COMPOSITION ON SEEDLING GROWTH OF TWO ATLANTIC FOREST TREE SPECIES
}

\author{
Joel Quintino de Oliveira Júnior ${ }^{1}$, Ana Caroline Rodrigues da Silva ${ }^{2}$, Paulo Victor Moreira da Silveira ${ }^{3}$, \\ Ederson da Conceição Jesus ${ }^{4}$, Marcos Gervasio Pereira*5
}

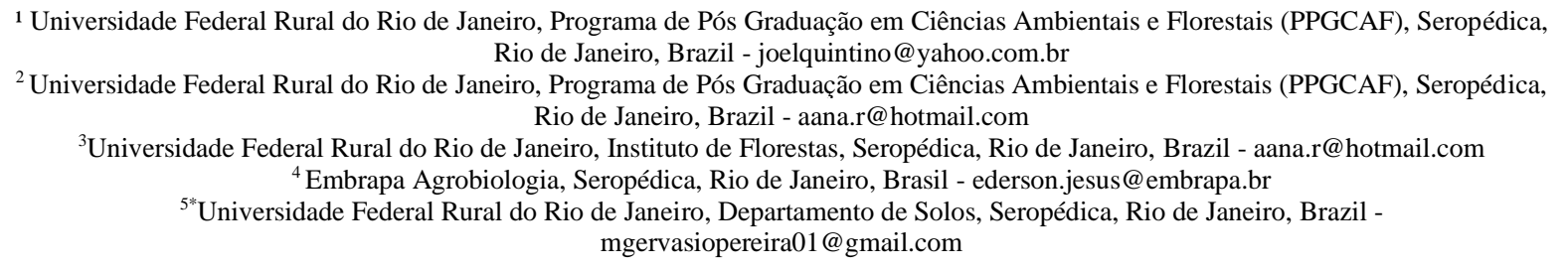

Received for publication: 14/08/2017 - Accepted for publication: 06/05/2019

\begin{abstract}
Resumo
Fungos micorrízicos e substratos na formação de mudas de duas espécies da Mata Atlântica. A busca por ferramentas biotecnológicas mais sustentáveis de recuperação ambiental cresceu nas últimas décadas, como os inoculantes microbianos. $\mathrm{O}$ qual tem sua eficiência ligada ao substrato utilizado para produção de mudas. $\mathrm{O}$ presente estudo objetivou avaliar as mudas de Pseubombax grandiflorum e Apuleia leiocarpa sob à inoculação com fungos micorrízicos arbusculares (FMA) em diferentes substratos e o seu crescimento. Os experimentos foram conduzidos em casa de vegetação com delineamento inteiramente casualizado em esquema fatorial $6 \mathrm{x}$ 2 (seis substratos e dois níveis de inoculação), com 12 tratamentos e seis repetições cada. As espécies apresentaram padrões de crescimento similares. Os melhores resultados foram utilizando-se as composições com esterco bovino e as maiores proporções de Cambissolo e Biomix, todos inoculados Fungos Micorrizicos. $\mathrm{O}$ resultado indica relação entre os fatores físicos e químicos do solo $(\mathrm{pH}$, dose ideal de fósforo e teor de nitrogênio) fornecidos pelos substratos, possibilitando a formação de cenário ideal para o melhor desenvolvimento das espécies e da simbiose micorrízica. Os melhores tratamentos para o crescimento de $P$. grandiflorum foram a combinação de $80 \%$ de terra do horizonte superficial de um Cambissolo com $20 \%$ de Biomix e FMA e a combinação de solo mais fósforo e FMA. Para a espécie A. leiocarpa os melhores tratamentos foram a combinação de $80 \%$ de solo com $20 \%$ de Biomix e FMA, $70 \%$ de solo com $30 \%$ de esterco e FMA e solo mais fósforo e FMA.

Palavras-chave: Micorriza, Inóculo, Simbiose, Produção de mudas.
\end{abstract}

\begin{abstract}
The search for more efficient biotechnological tools for forest regeneration has increased over the past decades. Mycorrhizal inoculation is a valuable strategy to promote seedling growth, but its efficiency depends on the substrate used. This study aimed to investigate the effects of arbuscular mycorrhizal fungal inoculation and different types of substrates on the growth of Pseudobombax grandiflorum and Apuleia leiocarpa seedlings. Experiments were carried out under greenhouse conditions in a completely randomized $6 \times 2$ factorial design (six substrates and two inoculation levels) with six replications of 12 treatments. Both species showed similar growth patterns. Plants grown in inoculated substrates composed of $70 \%$ Cambisol and $30 \%$ bovine manure or 80\% Cambisol and 20\% Biomix showed improved nutritional status. These substrates had good physical and chemical characteristics (pH and $\mathrm{P}$ and $\mathrm{N}$ levels), which favored plant development and mycorrhizal symbiosis. $P$. grandiflorum seedlings showed enhanced growth in inoculated substrate composed of $80 \%$ Cambisol and $20 \%$ Biomix and in inoculated Cambisol fertilized with P. For A. leiocarpa, the best results were observed for seedlings grown in inoculated substratescomposed of $80 \%$ Cambisol and 20\% Biomix, $70 \%$ Cambisol and 30\% bovine manure, or Cambisol fertilized with $\mathrm{P}$.

Keywords: Mycorrhiza; Inoculum, Symbiosis, Seedling production.
\end{abstract}

\section{INTRODUCTION}

Mycorrhiza is a symbiotic relationship between plants and fungi. Commonly found in association with plants, mycorrhizal fungi bring great benefits to their hosts (THIRKELL et al., 2016), including enhanced growth, nutrient uptake (in particular of low mobility compounds such as phosphorus), and resistance to biotic and abiotic stresses (Goetten et al., 2016; CHAER et al., 2011).

FLORESTA, Curitiba, PR, v. 49, n. 4, p. 623 - 632, out/dez 2019.

Oliveira Junior, J. Q. et.al.

ISSN eletrônico 1982-4688

DOI: $10.5380 /$ rf.v49 i4.54525 
Mycorrhizal fungi are used to restore degraded areas because they provide better conditions for plants to grow and compete for environmental resource. They act as root extensions, capturing resources (water and minerals) otherwise not accessible to plant roots, and contribute to soil aggregation by producing the glycoprotein glomalin. These beneficial fungi can also be used as biological indicators: the lower their density and diversity, the higher the degradation (SCORIZA et al., 2016). Because of their benefits, mycorrhizal fungi are an excellent tool for the production of forest seedlings. In particular, arbuscular mycorrhizal fungi stand out for their symbiotic efficiency and easy adaptation to the soil or substrate.

Substrate quality is an important factor for seedling development and establishment of mycorrhizal symbiosis. The substrate can positively or negatively affect nutritional and morphological aspects of young plants depending on its physical and chemical characteristics (Camara et al., 2017). Substrates must be chosen on the basis of plant type and economic factors. In general, substrates should have low density, high porosity, water holding capacity, and cation exchange capacity and be free of pests, pathogens, and weed seeds. The substrate is the main source of resources for developing seedlings and the intermediary between microorganism and host. A better understanding of fungal diversity and plant-soil-fungi relationships are needed.

Thus, the work tested the hypothesis that the change in substrate composition differently influences the quality of seedlings inoculated. The aim of this study was to evaluate the interaction of arbuscular mycorrhizal fungi with different substrates and their effects on the growth of seedlings of two Atlantic Forest species.

\section{MATERIAL AND METHODS \\ Experimental design}

Pseudobombax grandiflorum (Cav.) A. Robyns and Apuleia leiocarpa (Vogel) J.F.Macbr were grown under greenhouse conditions in two experimental periods, from December to April (124 days) and April to August (118 days) 2016. A completely randomized $6 \times 2$ factorial design (six substrates and two levels of inoculation) with six repetitions of 12 treatments was used. Inoculation levels consisted of inoculation or non-inoculation of substrates with arbuscular mycorrhizal fungi. Substrates in treatments $\mathrm{T}_{2}, \mathrm{~T}_{4}, \mathrm{~T}_{6}, \mathrm{~T}_{8}, \mathrm{~T}_{10}$, and $\mathrm{T}_{12}$ were inoculated with mycorrhizal fungi, whereas substrates in treatments $\mathrm{T}_{1}, \mathrm{~T}_{3}, \mathrm{~T}_{5}, \mathrm{~T}_{7}, \mathrm{~T}_{9}$, and $\mathrm{T}_{11}$ were not inoculated.

\section{Substrates}

The composition and chemical characteristics of the six substrates used in the experiment are shown in Table 1. The soil was classified as typical dystrophic Cambisol (Cambissolo Háplico). Soil samples were collected from the $10 \mathrm{~cm}$ depth. Substrate 6 (composed of Cambisol fertilized with phosphorous) was used in a prior evaluation of mycorrhizal dependence of plant species.

Table 1. Composition and chemical properties of substrates used in the experiments.

Tabela 1. Análise química dos substratos utilizados nos experimentos com as espécies P. grandiflorum e A. leiocarpa.

\begin{tabular}{|c|c|c|c|c|c|c|c|c|c|c|c|c|}
\hline \multirow{2}{*}{ Treatments } & \multirow{2}{*}{ Substrate } & \multirow{2}{*}{ Composition } & \multirow{2}{*}{$\begin{array}{c}\mathrm{pH} \text { in } \\
\mathrm{H}_{2} \mathrm{O}\end{array}$} & $\mathrm{Ca}$ & $\mathrm{Mg}$ & $\mathrm{Al}$ & $\mathrm{P}$ & $\mathrm{K}$ & \multirow{2}{*}{$\begin{array}{c}\mathrm{N} \\
\left(\mathrm{g} \mathrm{kg}^{-1}\right)\end{array}$} & $\mathrm{S}$ & CEC & \multirow{2}{*}{$\begin{array}{c}\mathrm{V} \\
(\%)\end{array}$} \\
\hline & & & & \multicolumn{3}{|c|}{$\left(\mathrm{cmol}_{\mathrm{c}} \mathrm{dm}^{-3}\right)$} & \multicolumn{2}{|c|}{$\left(\mathrm{mg} \mathrm{kg}^{-1}\right)$} & & \multicolumn{2}{|c|}{$\mathrm{cmol}_{\mathrm{c}} \mathrm{dm}^{-3}$} & \\
\hline $\mathrm{T}_{1}$ and $\mathrm{T}_{2}$ & 1 & $\begin{array}{c}60 \% \text { Cambisol, } 10 \% \\
\text { Biomix, } 10 \% \text { sand, } \\
20 \% \text { vermiculite }\end{array}$ & 7.08 & 3.0 & 12.11 & 0 & 14.35 & 35.41 & 1.1 & 15.23 & 16.34 & 93 \\
\hline $\mathrm{T}_{3}$ and $\mathrm{T}_{4}$ & 2 & $\begin{array}{c}80 \% \text { Cambisol, } 20 \% \\
\text { Biomix }\end{array}$ & 6.32 & 7.96 & 0.92 & 0 & 57.81 & 43.69 & 1.4 & 8.99 & 11.38 & 79 \\
\hline $\mathrm{T}_{5}$ and $\mathrm{T}_{6}$ & 3 & $\begin{array}{c}50 \% \text { Cambisol, } 30 \% \\
\text { Biomix, } 10 \% \text { sand, } \\
10 \% \text { vermiculite }\end{array}$ & 7.13 & 9.75 & 2.79 & 0 & 95.46 & 22.71 & 1.7 & 12.6 & 13.72 & 92 \\
\hline $\mathrm{T}_{7}$ and $\mathrm{T}_{8}$ & 4 & $\begin{array}{c}\text { 60\% Cambisol, } 40 \% \\
\text { Biomix }\end{array}$ & 6.81 & 11.17 & 2.19 & 0 & 83.46 & 77.48 & 1.7 & 13.56 & 15.99 & 85 \\
\hline $\mathrm{T}_{9}$ and $\mathrm{T}_{10}$ & 5 & $\begin{array}{c}70 \% \text { Cambisol, } 30 \% \\
\text { bovine manure }\end{array}$ & 6.61 & 5.82 & 1.97 & 0 & 89.39 & 84.56 & 3.1 & 8.01 & 10.34 & 78 \\
\hline $\mathrm{T}_{11}$ and $\mathrm{T}_{12}$ & 6 & $\begin{array}{c}\text { Cambisol fertilized } \\
\text { with phosphorus }\end{array}$ & 5.26 & 1.75 & 0.48 & 0.3 & 401.86 & 161.64 & 1.1 & 2.64 & 7.14 & 37 \\
\hline
\end{tabular}

$\mathrm{S}$, sum of bases $(\mathrm{Ca}, \mathrm{Mg}$, and $\mathrm{K})$; $\mathrm{CEC}$, cation exchange capacity at $\mathrm{pH} 7.0$, calculated by the formula $\mathrm{CEC}=\mathrm{S}+(\mathrm{H}+\mathrm{Al})$; $\mathrm{V}$, base saturation, calculated by the formula $\mathrm{V} \%=(100 \times \mathrm{S}) / \mathrm{T}$.

\section{Seed preparation}

A. leiocarpa and $P$. grandiflorum seeds were disinfected with $30 \% \mathrm{H}_{2} \mathrm{O}_{2}$ for 2 min, placed in Petri dishes containing filter paper and cotton, and incubated in a BOD germination chamber at $28{ }^{\circ} \mathrm{C}$ under constant light for 5 days until reaching $1 \mathrm{~cm}$ height. 


\section{Fungal inoculum}

A mixture of three arbuscular mycorrhizal fungal species was used: Dentiscutata heterogama (DHET A2, CNPAB002), Gigaspora margarita (GMAR A1, CNPAB001), and Rhizophagus clarus (RCLA A5, CNPAB005). Each starting container received $1 \mathrm{~g}$ of inoculum containing 17, 35, and 26 spores of D. heterogama, G. margarita, and $R$. clarus, respectively.

Uninoculated treatments received $100 \mathrm{~mL}$ of non-mycorrhizal microbiota as a control. Non-mycorrhizal microbiota was separated from $100 \mathrm{~g}$ of fungal inoculum by suspension in $1 \mathrm{~L}$ of distilled water, followed by filtering through a $50 \mathrm{~mm}$ filter paper.

\section{Experiments}

Starting containers were constructed using $280 \mathrm{~mL}$ plastic vials and $700 \mathrm{~mL}$ disposable plastic cups. Containers were filled with $1 \mathrm{~kg}$ of substrate. Prior to the experiment, A. leiocarpa seeds were scarified by immersion in sulfuric acid for $15 \mathrm{~min}$ to overcome dormancy. Plants were watered daily to $70 \%$ of the field capacity by spray irrigation. P. grandiflorum was evaluated for 124 days and A. leiocarpa for 118 days.

\section{Sampling procedures}

After the experimental period, seedlings were collected and separated into shoots and roots. From three replicates, $0.5 \mathrm{~g}$ of fine roots were used for evaluation of mycorrhizal colonization (KOSKE and GEMMA, 1989). Fungal colonization was evaluated by the gridline intersect method, according to Giovannetti and Mosse (1980). Root samples from the other three replicates were dried in a forced-air oven $\left(65{ }^{\circ} \mathrm{C}, 72 \mathrm{~h}\right)$ and weighed for determination of root dry weight (RDW). Samples from all replicates were used to determine shoot dry weight (SDW). After SDW determination, shoots were crushed and subjected to sulfuric acid digestion for determination of N, P, and K. P levels were assessed by colorimetric analysis, K by flame photometry (TEDESCO, et al. 1995), and $\mathrm{N}$ by the modified Kjeldahl method.

\section{Variables}

The variables evaluated were SDW, RDW, total dry weight (TDW), height (H), diameter (D), and mycorrhizal colonization percentage. Plant height was measured from the collet to the terminal bud. The following indices were determined to assess seedling quality: H/D, H/SDW, SDW/RDW, and the Dickson quality index (DQI) (DICKSON et al., 1960).

\section{Data analysis}

Data were first Box-Cox transformed to meet normality assumptions via the Shapiro-Wilk test. Transformed data were subjected to analysis of variance. Means were compared using the Scott-Knott test ( $p<$ 0.05). Statistical analyses were carried out using Sisvar (FERREIRA, 2011). Principal component analysis (PCA) was performed based on the correlation matrix using the $\mathrm{R}$ program with the vegan package. The number of extracted components was chosen on the basis of the cumulative variance (minimum of $70 \%$ ).

\section{RESULTS}

\section{Seedling response to inoculation}

Inoculation increased SDW and TDW in both species, regardless of the substrate used. TDW was $300 \%$ higher in $P$. grandiflorum grown in inoculated Cambisol fertilized with $\mathrm{P}\left(\mathrm{T}_{12}\right)$ than in uninoculated substrate $\left(\mathrm{T}_{11}\right)$ (Table 2). For A. leiocarpa grown in P-fertilized Cambisol, inoculation promoted a $400 \%$ increase in TDW. Even in the presence of high $\mathrm{P}$ concentrations $\left(650 \mathrm{mg} \mathrm{kg}^{-1}\right.$ soil), uninoculated plants did not increase in height, diameter, or SDW $\left(\mathrm{T}_{11}\right)$, evidence of the plants' dependence on mycorrhizal fungi (Table 2)

The $\mathrm{T}_{4}$ treatment $(80 \%$ Cambisol $+20 \%$ Biomix + mycorrhizal inoculum) resulted in the highest biomass gain for $P$. grandiflorum. For, A. leiocarpa the highest biomass gain was obtained with $\mathrm{T}_{12}(\mathrm{Cambisol}+\mathrm{P}+$ mycorrhizal inoculum) but the highest TDW value was obtained with $\mathrm{T}_{10}(70 \%$ Cambisol $+30 \%$ bovine manure + mycorrhizal inoculum) (Table 3).

Mycorrhizal colonization in P. grandiflorum ranged from $11 \%$ in $\mathrm{T}_{10}(70 \%$ Cambisol $+30 \%$ bovine manure) to $24 \%$ in $\mathrm{T}_{12}($ Cambisol $+\mathrm{P})$, varying according to the concentration of $\mathrm{P}$ in the soil (Table 2). In $A$. leiocarpa, mycorrhizal colonization varied from $18 \%$ in $\mathrm{T}_{10}$ to $33 \%$ in $\mathrm{T}_{12}$ (Table 3 ).

$P$. grandiflorum seedling height was highest in $\mathrm{T}_{8}$ and $\mathrm{T}_{9}(70 \%$ Cambisol $+30 \%$ manure, with and without inoculation), whereas A. leiocarpa height was highest in $\mathrm{T}_{3}$ and $\mathrm{T}_{4}(80 \%$ Cambisol $+20 \%$ Biomix, with and without inoculation) and $\mathrm{T}_{11}$ and $\mathrm{T}_{12}$ (Cambisol $+\mathrm{P}$, with and without inoculation) (Table 3 ).

$P$. grandiflorum diameter was largest in $\mathrm{T}_{8}$ and $\mathrm{T}_{9}$ (Table 2). A. leiocarpa, in contrast, showed no variation in diameter between treatments (Table 3). 
Table 2. Effects of substrate and mycorrhizal fungal inoculation on Pseudobombax grandiflorum seedling growth. Tabela 2. Efeito de diferentes tipos de substrato e da inoculação com fungos micorrízicos no crescimento da espécie $P$. grandiflorum.

\begin{tabular}{|c|c|c|c|c|c|c|}
\hline Treatment & RDW (g) & SDW (g) & TDW (g) & Diameter (mm) & Height $(\mathrm{cm})$ & $\mathrm{M}(\%)$ \\
\hline $\mathrm{T}_{1}$ & $0.48 \mathrm{c}$ & $0.67 \mathrm{c}$ & $1.15 \mathrm{c}$ & $5.09 \mathrm{c}$ & $8.35 \mathrm{c}$ & \\
\hline $\mathrm{T}_{2}$ & $0.64 \mathrm{c}$ & $0.91 \mathrm{~b}$ & $1.55 \mathrm{~b}$ & $5.06 \mathrm{c}$ & $7.33 \mathrm{c}$ & $18 \mathrm{a}$ \\
\hline $\mathrm{T}_{3}$ & $0.57 \mathrm{c}$ & $0.63 \mathrm{c}$ & $1.20 \mathrm{c}$ & $6.47 \mathrm{~b}$ & $9.70 \mathrm{c}$ & \\
\hline $\mathrm{T}_{4}$ & $1.31 \mathrm{a}$ & $1.31 \mathrm{a}$ & $2.62 \mathrm{a}$ & $6.85 \mathrm{~b}$ & $11.03 \mathrm{~b}$ & $20 \mathrm{a}$ \\
\hline $\mathrm{T}_{5}$ & $0.42 \mathrm{c}$ & $0.59 \mathrm{c}$ & $1.01 \mathrm{c}$ & $5.01 \mathrm{c}$ & $7.86 \mathrm{c}$ & \\
\hline $\mathrm{T}_{6}$ & $0.51 \mathrm{c}$ & $0.84 \mathrm{~b}$ & $1.35 \mathrm{c}$ & $4.92 \mathrm{c}$ & $7.34 \mathrm{c}$ & $17 \mathrm{a}$ \\
\hline $\mathrm{T}_{7}$ & $0.56 \mathrm{c}$ & $0.80 \mathrm{~b}$ & $1.36 \mathrm{c}$ & $3.95 \mathrm{c}$ & $6.21 \mathrm{c}$ & \\
\hline $\mathrm{T}_{8}$ & $0.88 \mathrm{~b}$ & $0.93 \mathrm{~b}$ & $1.81 \mathrm{~b}$ & $4.77 \mathrm{c}$ & $7.51 \mathrm{c}$ & $13 \mathrm{~b}$ \\
\hline $\mathrm{T}_{9}$ & $0.11 \mathrm{~d}$ & $0.52 \mathrm{~d}$ & $0.63 \mathrm{~d}$ & $7.56 \mathrm{a}$ & $13.72 \mathrm{a}$ & \\
\hline $\mathrm{T}_{10}$ & $0.85 \mathrm{~b}$ & $0.78 \mathrm{~b}$ & $1.63 \mathrm{~b}$ & $8.27 \mathrm{a}$ & $14.83 \mathrm{a}$ & $11 \mathrm{~b}$ \\
\hline $\mathrm{T}_{11}$ & $0.26 \mathrm{~d}$ & $0.38 \mathrm{~d}$ & $0.64 \mathrm{~d}$ & $6.04 \mathrm{~b}$ & $10.38 \mathrm{~b}$ & \\
\hline $\mathrm{T}_{12}$ & $0.71 \quad \mathrm{c}$ & $1.23 \mathrm{a}$ & $1.94 \mathrm{~b}$ & $6.41 \mathrm{~b}$ & $10.63 \mathrm{~b}$ & $24 \mathrm{a}$ \\
\hline \multicolumn{7}{|l|}{$F$-test } \\
\hline Substrate & 0 & 0 & 0 & & & \\
\hline Fungi & 0 & 0 & 0 & & & \\
\hline Interaction & 0.01 & 0.02 & 0.02 & & & \\
\hline $\mathrm{CV}(\%)$ & 50.64 & 19.24 & 26.3 & 15.34 & 20.33 & 21.77 \\
\hline
\end{tabular}

Means in a column followed by different letters differ significantly by the Scott-Knott test $(p<0.05)$. $\mathrm{T}_{1}, 60 \% \mathrm{Cambisol}+10 \% \mathrm{Biomix}+10 \%$ sand $+20 \%$ vermiculite, uninoculated; $\mathrm{T}_{2}, 60 \%$ Cambisol $+10 \%$ Biomix $+10 \%$ sand $+20 \%$ vermiculite + mycorrhizal inoculum; $\mathrm{T}_{3}, 80 \%$ Cambisol $+20 \%$ Biomix, uninoculated $\mathrm{T}_{4}, 80 \%$ Cambisol $+20 \%$ Biomix + mycorrhizal inoculum; $\mathrm{T}_{5}, 50 \% \mathrm{Cambisol}+30 \% \mathrm{Biomix}+10 \%$ sand $+10 \%$ vermiculite, uninoculated; $\mathrm{T}_{6}, 50 \%$ Cambisol $+30 \%$ Biomix $+10 \%$ sand $+10 \%$ vermiculite + mycorrhizal inoculum; $\mathrm{T}_{7}, 60 \%$ Cambisol $+40 \%$ Biomix, uninoculated $; \mathrm{T}_{8}, 60 \%$ Cambisol $+40 \%$ Biomix + mycorrhizal inoculum; $\mathrm{T}_{9}, 70 \%$ Cambisol $+30 \%$ bovine manure, uninoculated; $\mathrm{T}_{10}, 70 \%$ Cambisol $+30 \%$ bovine manure + mycorrhizal inoculum; T11, $100 \%$ Cambisol fertilized with phosphorus, uninoculated; $\mathrm{T}_{12}, 100 \%$ Cambisol fertilized with phosphorus + mycorrhizal inoculum; RDW, root dry weight; SDW, shoot dry weight; TDW, total dry weight; $\mathrm{M}$, mycorrhizal colonization; $\mathrm{CV}$, coefficient of variation.

Table 3. Effects of substrate and mycorrhizal fungal inoculation on Apuleia leiocarpa seedling growth.

Tabela 3. Efeito de diferentes tipos de substrato e da inoculação com fungos micorrízicos no crescimento da espécie A. leiocarpa.

\begin{tabular}{|c|c|c|c|c|c|c|}
\hline Treatment & RDW (g) & $\mathrm{SDW}(\mathrm{g})$ & TDW (g) & Diameter $(\mathrm{mm})$ & Height $(\mathrm{cm})$ & $\mathrm{M}(\%)$ \\
\hline $\mathrm{T}_{1}$ & $0.23 \mathrm{c}$ & $0.33 \mathrm{c}$ & $0.57 \mathrm{~b}$ & $1.39 \mathrm{a}$ & $7.01 \mathrm{~b}$ & \\
\hline $\mathrm{T}_{2}$ & $0.26 \mathrm{~b}$ & $0.26 \mathrm{c}$ & $0.52 \mathrm{c}$ & $1.32 \mathrm{a}$ & $7.15 \mathrm{~b}$ & $24 \mathrm{~b}$ \\
\hline $\mathrm{T}_{3}$ & $0.16 \mathrm{~d}$ & $0.27 \quad b$ & $0.43 \mathrm{~d}$ & $1.37 \mathrm{a}$ & 9.48 a & \\
\hline $\mathrm{T}_{4}$ & $0.22 \mathrm{c}$ & $0.66 \mathrm{a}$ & 0.88 a & $1.58 \mathrm{a}$ & $11.36 \mathrm{a}$ & 36 a \\
\hline $\mathrm{T}_{5}$ & $0.22 \mathrm{c}$ & $0.39 \mathrm{~b}$ & $0.61 \mathrm{~b}$ & $1.30 \mathrm{a}$ & $6.35 \mathrm{~b}$ & \\
\hline $\mathrm{T}_{6}$ & $0.31 \mathrm{~b}$ & $0.43 \mathrm{a}$ & $0.75 \mathrm{~b}$ & $1.26 \mathrm{a}$ & $6.46 \mathrm{~b}$ & $25 \mathrm{~b}$ \\
\hline $\mathrm{T}_{7}$ & $0.15 \mathrm{c}$ & $0.33 \mathrm{c}$ & $0.48 \mathrm{~d}$ & $1.29 \mathrm{a}$ & $5.15 \mathrm{~b}$ & \\
\hline $\mathrm{T}_{8}$ & $0.28 \mathrm{~b}$ & $0.47 \quad b$ & $0.75 \mathrm{~b}$ & $1.31 \mathrm{a}$ & $6.41 \mathrm{~b}$ & $21 \mathrm{~b}$ \\
\hline $\mathrm{T}_{9}$ & $0.14 \mathrm{~d}$ & $0.23 \mathrm{c}$ & $0.37 \mathrm{~d}$ & $1.36 \mathrm{a}$ & $6.76 \mathrm{~b}$ & \\
\hline $\mathrm{T}_{10}$ & $0.23 \mathrm{c}$ & $0.68 \mathrm{a}$ & $0.91 \mathrm{a}$ & $1.40 \mathrm{a}$ & $6.78 \mathrm{~b}$ & $18 \mathrm{~b}$ \\
\hline $\mathrm{T}_{11}$ & $0.09 \mathrm{~d}$ & $0.15 \mathrm{~d}$ & $0.24 \mathrm{~d}$ & $1.30 \mathrm{a}$ & $9.56 \mathrm{a}$ & \\
\hline $\mathrm{T}_{12}$ & $0.45 \mathrm{a}$ & $0.58 \quad \mathrm{a}$ & $1.03 \mathrm{a}$ & $1.53 \mathrm{a}$ & $10.16 \mathrm{a}$ & 33 a \\
\hline \multicolumn{7}{|l|}{$F$-test } \\
\hline Substrate & 0.35 & 0 & 0.002 & & & \\
\hline Fungi & 0 & 0 & 0 & & & \\
\hline Interaction & 0 & 0.16 & 0 & & & \\
\hline $\mathrm{CV}(\%)$ & 39.16 & 41.07 & 36.33 & 14.29 & 21.88 & 24.15 \\
\hline
\end{tabular}

Means in a column followed by different letters differ significantly by the Scott-Knott test $(p<0.05)$. $\mathrm{T}_{1}, 60 \%$ Cambisol $+10 \%$ Biomix $+10 \%$ sand $+20 \%$ vermiculite, uninoculated; $\mathrm{T}_{2}, 60 \%$ Cambisol $+10 \%$ Biomix $+10 \%$ sand $+20 \%$ vermiculite + mycorrhizal inoculum; $\mathrm{T}_{3}, 80 \%$ Cambisol $+20 \%$ Biomix, uninoculated; $\mathrm{T}_{4}, 80 \%$ Cambisol $+20 \%$ Biomix + mycorrhizal inoculum; $\mathrm{T}_{5}, 50 \%$ Cambisol $+30 \%$ Biomix $+10 \%$ sand $+10 \%$ vermiculite, uninoculated; $\mathrm{T}_{6}, 50 \%$ Cambisol $+30 \%$ Biomix $+10 \%$ sand $+10 \%$ vermiculite + mycorrhizal inoculum; $\mathrm{T}_{7}, 60 \%$ Cambisol $+40 \%$ Biomix, uninoculated; $\mathrm{T}_{8}, 60 \%$ Cambisol $+40 \%$ Biomix + mycorrhizal inoculum; $\mathrm{T}_{9}, 70 \%$ Cambisol $+30 \%$ bovine manure, uninoculated; $\mathrm{T}_{10}, 70 \%$ Cambisol $+30 \%$ bovine manure + mycorrhizal inoculum; T11, 100\% Cambisol fertilized with phosphorus, uninoculated; $\mathrm{T}_{12}, 100 \%$ Cambisol fertilized with phosphorus + mycorrhizal inoculum; RDW, root dry weight; SDW, shoot dry weight; TDW, total dry weight; $\mathrm{M}$, mycorrhizal colonization; $\mathrm{CV}$, coefficient of variation. 


\section{Seedling quality}

There was no significant difference in H/D index between treatments for $P$. grandiflorum (Table 4). For A. leiocarpa, significantly higher $\mathrm{H} / \mathrm{D}$ indices were obtained in $\mathrm{T}_{3}$ and $\mathrm{T}_{4}$ (uninoculated and inoculated substratescomposed of $80 \%$ Cambisol $+20 \%$ Biomix) and $\mathrm{T}_{10}$ and $\mathrm{T}_{11}$ (uninoculated and inoculated Cambisol fertilized with P) (Table 5).

P. grandiflorum seedlings showed a four times higher SDW/RDW index when grown in uninoculated Cambisol and bovine manure $\left(\mathrm{T}_{9}\right)$ (Table 4$)$. This substrate affected plant development by promoting shoot growth to the detriment of root growth. In contrast, A. leiocarpa seedlings showed altered development in inoculated substrate composed of $80 \%$ Cambisol and 20\% Biomix $\left(\mathrm{T}_{4}\right)$, with an SDW/RDW index three times higher than that obtained in inoculated substrate composed of 60\% Cambisol, 10\% Biomix, 20\% sand, and 10\% vermiculite $\left(\mathrm{T}_{2}\right)$.

The highest DQI for $P$. grandiflorum were obtained in T4 $(80 \%$ Cambisol $+20 \%$ Biomix + mycorrhizal inoculum) (1.01), T8 (60\% Cambisol + 40\% Biomix + mycorrhizal inoculum) (0.69), $\mathrm{T}_{10}$ (70\% Cambisol $+30 \%$ bovine manure + mycorrhizal inoculum) $(0.60)$, and $\mathrm{T}_{12}$ (Cambisol fertilized with phosphorus + mycorrhizal inoculum) (0.57) (Table 4). DQI values for A. leiocarpa were generally low; the best DQI were observed in $\mathrm{T}_{6}$ $(50 \%$ Cambisol $+30 \%$ Biomix $+10 \%$ sand $+10 \%$ vermiculite + mycorrhizal inoculum $)(0.12), \mathrm{T}_{10}(70 \%$ Cambisol $+30 \%$ bovine manure + mycorrhizal inoculum) $(0.12)$, and T12 (Cambisol fertilized with phosphorus + mycorrhizal inoculum) (0.13) (Table 5).

Table 4. Quality parameters of Pseudobombax grandiflorum seedlings grown in inoculated and uninoculated substrates.

Tabela 4. Índice de qualidade das mudas de $P$. grandiflorum produzidas em diferentes tipos de substrato e inoculadas com fungos micorrízicos.

\begin{tabular}{|c|c|c|c|c|c|c|}
\hline \multirow{2}{*}{ Treatment } & \multicolumn{6}{|c|}{ Index } \\
\hline & $\mathrm{H} / \mathrm{D}$ & & H/SDW & & SDW/RDW & DQI \\
\hline $\mathrm{T}_{1}$ & 1.64 & $\mathrm{a}$ & 12.39 & c & $1.40 \mathrm{~b}$ & $0.38 \mathrm{c}$ \\
\hline $\mathrm{T}_{2}$ & 1.45 & $\mathrm{a}$ & 8.09 & $\mathrm{~d}$ & $1.41 \mathrm{~b}$ & $0.54 \mathrm{c}$ \\
\hline $\mathrm{T}_{3}$ & 1.50 & $\mathrm{a}$ & 15.34 & b & $1.12 \mathrm{~b}$ & $0.46 \mathrm{c}$ \\
\hline $\mathrm{T}_{4}$ & 1.61 & $\mathrm{a}$ & 8.44 & $\mathrm{~d}$ & $0.99 \mathrm{~b}$ & $1.01 \mathrm{a}$ \\
\hline $\mathrm{T}_{5}$ & 1.57 & $\mathrm{a}$ & 13.32 & c & $1.42 \mathrm{~b}$ & $0.34 \mathrm{c}$ \\
\hline $\mathrm{T}_{6}$ & 1.49 & $\mathrm{a}$ & 8.76 & d & $1.65 \mathrm{~b}$ & $0.43 \mathrm{c}$ \\
\hline $\mathrm{T}_{7}$ & 1.72 & $\mathrm{a}$ & 12.91 & $\mathrm{c}$ & $1.43 \mathrm{~b}$ & $0.43 \mathrm{c}$ \\
\hline $\mathrm{T}_{8}$ & 1.57 & $\mathrm{a}$ & 8.04 & $\mathrm{~d}$ & $1.06 \mathrm{~b}$ & $0.69 \mathrm{~b}$ \\
\hline $\mathrm{T}_{9}$ & 1.81 & $\mathrm{a}$ & 26.46 & $\mathrm{a}$ & $4.56 \mathrm{a}$ & $0.10 \mathrm{~d}$ \\
\hline $\mathrm{T}_{10}$ & 1.79 & $\mathrm{a}$ & 19.09 & b & $0.91 \mathrm{~b}$ & $0.60 \mathrm{~b}$ \\
\hline $\mathrm{T}_{11}$ & 1.57 & $\mathrm{a}$ & 16.16 & b & $1.48 \mathrm{~b}$ & $0.21 \mathrm{~d}$ \\
\hline $\mathrm{T}_{12}$ & 1.66 & $\mathrm{a}$ & 8.65 & $\mathrm{~d}$ & $1.73 \mathrm{~b}$ & $0.57 \mathrm{~b}$ \\
\hline $\mathrm{CV}(\%)$ & 6.84 & & 40.54 & & 57.89 & 46.63 \\
\hline
\end{tabular}

Means in a column followed by different letters differ significantly by the Scott-Knott test $(p<0.05)$. $\mathrm{T}_{1}, 60 \% \mathrm{Cambisol}+10 \% \mathrm{Biomix}+10 \%$ sand $+20 \%$ vermiculite, uninoculated; $\mathrm{T}_{2}, 60 \%$ Cambisol $+10 \%$ Biomix $+10 \%$ sand $+20 \%$ vermiculite + mycorrhizal inoculum; $\mathrm{T}_{3}, 80 \%$ Cambisol $+20 \%$ Biomix, uninoculated $\mathrm{T}_{4}, 80 \%$ Cambisol $+20 \%$ Biomix + mycorrhizal inoculum; $\mathrm{T}_{5}, 50 \% \mathrm{Cambisol}+30 \% \mathrm{Biomix}+10 \%$ sand $+10 \%$ vermiculite, uninoculated; $\mathrm{T}_{6}, 50 \%$ Cambisol $+30 \%$ Biomix $+10 \%$ sand $+10 \%$ vermiculite + mycorrhizal inoculum; $\mathrm{T}_{7}, 60 \%$ Cambisol $+40 \%$ Biomix, uninoculated $\mathrm{T}_{8}, 60 \%$ Cambisol $+40 \%$ Biomix + mycorrhizal inoculum; $\mathrm{T}_{9}, 70 \%$ Cambisol $+30 \%$ bovine manure, uninoculated; $\mathrm{T}_{10}, 70 \%$ Cambisol $+30 \%$ bovine manure + mycorrhizal inoculum; $\mathrm{T} 11$, Cambisol fertilized with phosphorus, uninoculated; $\mathrm{T}_{12}$, Cambisol fertilized with phosphorus + mycorrhizal inoculum; CV, coefficient of variation; H/D, ratio of plant height to diameter; H/SDW, ratio of plant height to shoot dry weight; SDW/RDW, ratio of shoot dry weight to root dry weight; DQI, Dickson quality index, calculated according to Dickson (1960).

Table 5. Quality parameters of Apuleia leiocarpa seedlings grown in inoculated and uninoculated substrates.

Tabela 5. Índice de qualidade das mudas de A. leiocarpa produzidas em diferentes tipos de substrato e inoculadas com fungos micorrízicos.

FLORESTA, Curitiba, PR, v. 49, n. 4, p. 623 - 632, out/dez 2019.

Oliveira Junior, J. Q. et.al.

ISSN eletrônico 1982-4688

DOI: $10.5380 /$ rf.v49 i4.54525 


\begin{tabular}{|c|c|c|c|c|c|c|}
\hline \multirow{3}{*}{$\begin{array}{c}\text { Treatment } \\
\mathrm{T}_{1}\end{array}$} & \multicolumn{6}{|c|}{ Index } \\
\hline & H/D & \multicolumn{2}{|l|}{ H/SDW } & SDW/RDW & \multicolumn{2}{|l|}{ DQI } \\
\hline & $5.04 \mathrm{~b}$ & 21.24 & $\mathrm{~b}$ & $1.43 \mathrm{~b}$ & 0.09 & b \\
\hline $\mathrm{T}_{2}$ & $5.42 \mathrm{~b}$ & 27.50 & $\mathrm{~b}$ & $1.00 \mathrm{c}$ & 0.08 & b \\
\hline $\mathrm{T}_{3}$ & $6.92 \mathrm{a}$ & 35.11 & $\mathrm{~b}$ & $1.69 \mathrm{~b}$ & 0.05 & $\mathrm{c}$ \\
\hline $\mathrm{T}_{4}$ & $7.19 \mathrm{a}$ & 17.21 & $\mathrm{c}$ & $3.00 \mathrm{a}$ & 0.09 & $\mathrm{~b}$ \\
\hline $\mathrm{T}_{5}$ & $4.88 \mathrm{~b}$ & 16.28 & $\mathrm{c}$ & $1.77 \mathrm{~b}$ & 0.09 & $\mathrm{~b}$ \\
\hline $\mathrm{T}_{6}$ & $5.13 \mathrm{~b}$ & 15.02 & $\mathrm{c}$ & $1.39 \mathrm{~b}$ & 0.12 & $\mathrm{a}$ \\
\hline $\mathrm{T}_{7}$ & $3.99 \mathrm{~b}$ & 15.61 & $\mathrm{c}$ & $2.20 \mathrm{~b}$ & 0.08 & b \\
\hline $\mathrm{T}_{8}$ & $4.89 \mathrm{~b}$ & 13.64 & $\mathrm{c}$ & $1.68 \mathrm{~b}$ & 0.11 & B \\
\hline $\mathrm{T}_{9}$ & $4.97 \mathrm{~b}$ & 29.39 & $\mathrm{~b}$ & $1.64 \mathrm{~b}$ & 0.06 & $\mathrm{c}$ \\
\hline $\mathrm{T}_{10}$ & $4.84 \mathrm{~b}$ & 9.97 & $\mathrm{~d}$ & $2.96 \mathrm{a}$ & 0.12 & $\mathrm{a}$ \\
\hline $\mathrm{T}_{11}$ & $7.35 \quad \mathrm{a}$ & 63.73 & $\mathrm{a}$ & $1.67 \mathrm{~b}$ & 0.03 & $\mathrm{c}$ \\
\hline $\mathrm{T}_{12}$ & $6.64 \quad \mathrm{a}$ & 17.52 & $\mathrm{c}$ & $1.29 \mathrm{~b}$ & 0.13 & $\mathrm{a}$ \\
\hline $\mathrm{CV}(\%)$ & 19.8 & 62.16 & & 34.19 & 35.67 & \\
\hline
\end{tabular}

Means in a column followed by different letters differ significantly by the Scott-Knott test $(p<0.05) . \mathrm{T}_{1}, 60 \% \mathrm{Cambisol}+10 \%$ Biomix $+10 \%$ sand $+20 \%$ vermiculite, uninoculated; $\mathrm{T}_{2}, 60 \%$ Cambisol $+10 \%$ Biomix $+10 \%$ sand $+20 \%$ vermiculite + mycorrhizal inoculum; $\mathrm{T}_{3}, 80 \%$ Cambisol $+20 \%$ Biomix, uninoculated; $\mathrm{T}_{4}, 80 \%$ Cambisol $+20 \%$ Biomix + mycorrhizal inoculum; $\mathrm{T}_{5}, 50 \% \mathrm{Cambisol}+30 \% \mathrm{Biomix}+10 \%$ sand $+10 \%$ vermiculite, uninoculated; $\mathrm{T}_{6}, 50 \%$ Cambisol $+30 \%$ Biomix $+10 \%$ sand $+10 \%$ vermiculite + mycorrhizal inoculum; $\mathrm{T}_{7}, 60 \%$ Cambisol $+40 \%$ Biomix, uninoculated $\mathrm{T}_{8}, 60 \%$ Cambisol $+40 \%$ Biomix + mycorrhizal inoculum; $\mathrm{T}_{9}, 70 \%$ Cambisol $+30 \%$ bovine manure, uninoculated; $\mathrm{T}_{10}, 70 \%$ Cambisol $+30 \%$ bovine manure + mycorrhizal inoculum; $\mathrm{T} 11$, Cambisol fertilized with phosphorus, uninoculated; $\mathrm{T}_{12}$, Cambisol fertilized with phosphorus + mycorrhizal inoculum; CV, coefficient of variation; H/D, ratio of plant height to diameter; H/SDW, ratio of plant height to shoot dry weight; SDW/RDW, ratio of shoot dry weight to root dry weight; DQI, Dickson quality index, calculated according to Dickson (1960).

PCA of $P$. grandiflorum data (Figure 1) identified two principal components, which together explained $90.83 \%$ of the variance in the dataset. Principal component 1 (PC1) was highly correlated with biomass parameters (RDW and SDW), whereas principal component 2 (PC2) was strongly correlated with growth parameters (height and diameter). Treatments could be divided into two groups according to their position in the PCA biplot (Figure 1): inoculated treatments are located on the right-hand side of the graph, whereas uninoculated treatments are plotted on the left-hand side. It can also be seen that the best treatments for $P$. grandiflorum were $\mathrm{T}_{4}(80 \%$ Cambisol $+20 \%$ Biomix + mycorrhizal inoculum) and $\mathrm{T}_{12}$ (Cambisol fertilized with $\mathrm{P}+$ mycorrhizal inoculum), confirming the beneficial effect of mycorrhizal inoculation.

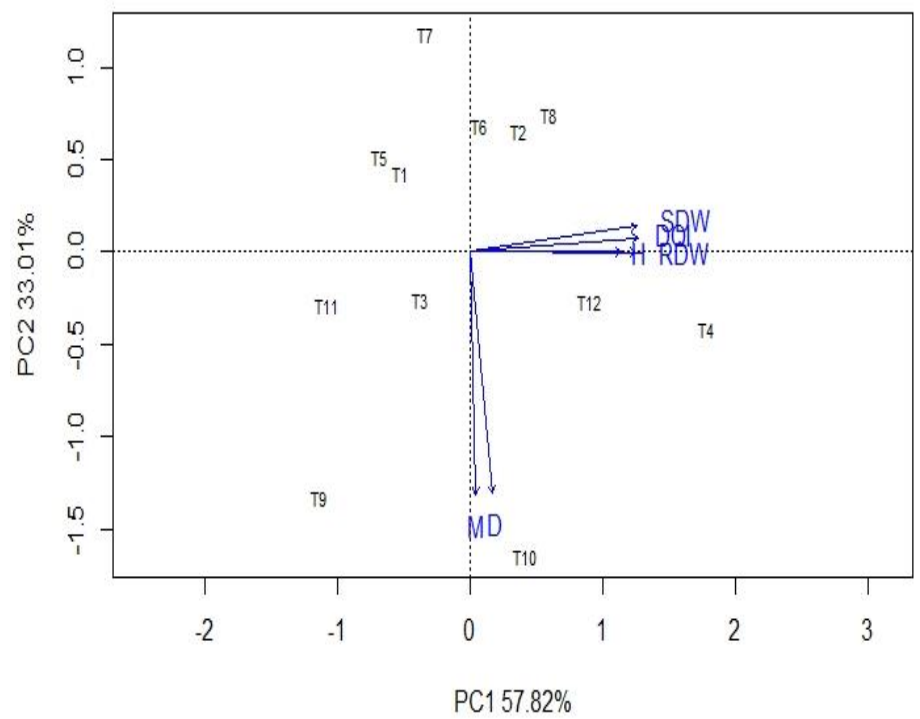

Figure 1. Principal component analysis of biomass and growth data of Pseudobombax grandiflorum seedlings grown in inoculated and uninoculated substrates. 
Figura 1. Análise de Componentes Principais das variáveis de biomassa e crescimento mensuradas em experimento com a espécie $P$. grandiflorum, discriminando tratamentos com e sem inoculação.

Data are the mean of six replicate determinations. D, diameter; H, height; SDW, shoot dry weight; RDW, root dry weight; DQI, Dickson quality index; $\mathrm{M}$, mycorrhizal colonization; $\mathrm{T}_{1}, 60 \%$ Cambisol $+10 \%$ Biomix $+10 \%$ sand $+20 \%$ vermiculite, uninoculated; $\mathrm{T}_{2}, 60 \%$ Cambisol $+10 \%$ Biomix $+10 \%$ sand $+20 \%$ vermiculite + mycorrhizal inoculum; $\mathrm{T}_{3}, 80 \%$ Cambisol $+20 \%$ Biomix, uninoculated; $\mathrm{T}_{4}, 80 \%$ Cambisol + $20 \%$ Biomix + mycorrhizal inoculum; $\mathrm{T}_{5}, 50 \%$ Cambisol $+30 \%$ Biomix $+10 \%$ sand $+10 \%$ vermiculite, uninoculated; $\mathrm{T}_{6}, 50 \%$ Cambisol + $30 \%$ Biomix $+10 \%$ sand $+10 \%$ vermiculite + mycorrhizal inoculum; $\mathrm{T}_{7}, 60 \%$ Cambisol $+40 \%$ Biomix, uninoculated; $\mathrm{T}_{8}, 60 \%$ Cambisol + $40 \%$ Biomix + mycorrhizal inoculum; $\mathrm{T}_{9}, 70 \%$ Cambisol $+30 \%$ bovine manure, uninoculated; $\mathrm{T}_{10}, 70 \%$ Cambisol $+30 \%$ bovine manure + mycorrhizal inoculum; T11, Cambisol fertilized with phosphorus, uninoculated; $\mathrm{T}_{12}$, Cambisol fertilized with phosphorus + mycorrhizal inoculum.

PCA of A. leiocarpa data (Figure 2) extracted two principal components, which accounted for $86.81 \%$ of the total variance. Uninoculated treatments also appeared to the left and inoculated treatments to the right of the biplot. The best treatments for A. leiocarpa were $\mathrm{T}_{4}(80 \%$ Cambisol $+20 \%$ Biomix + mycorrhizal inoculum $), \mathrm{T}_{10}$ ( $70 \%$ Cambisol $+30 \%$ bovine manure + mycorrhizal inoculum $)$, and $\mathrm{T}_{12}(\mathrm{Cambisol}$ fertilized with $\mathrm{P}+$ mycorrhizal inoculum).

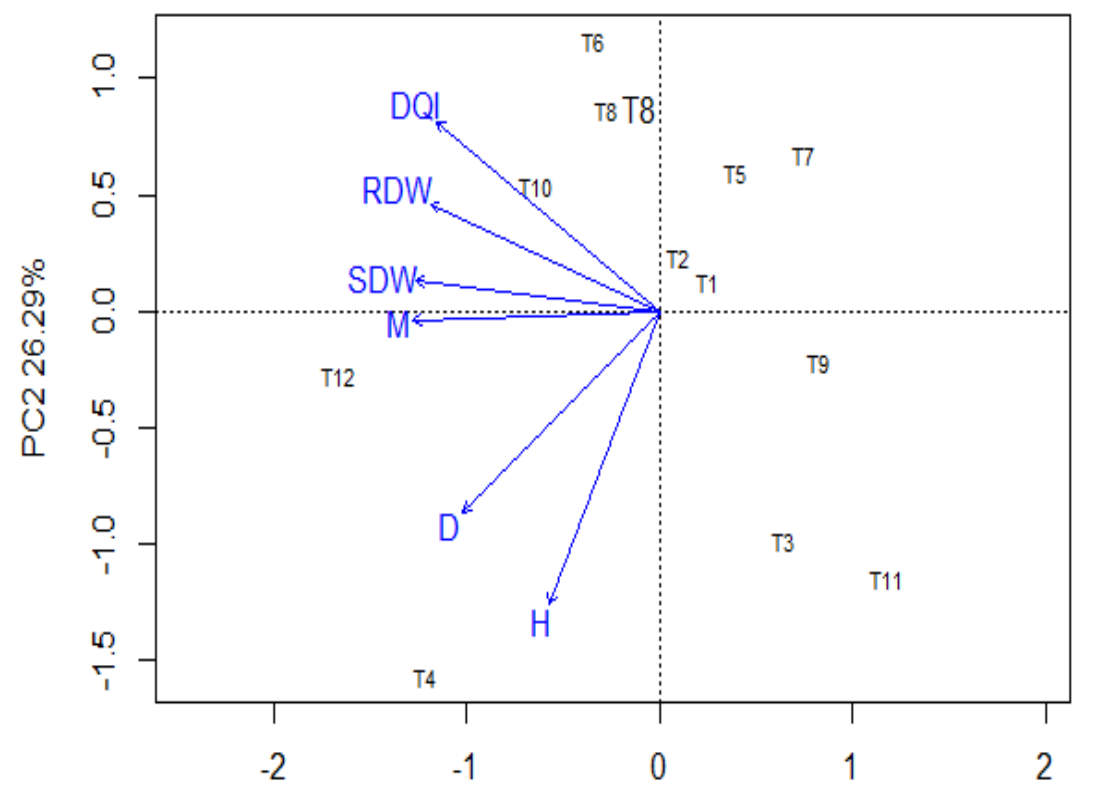

PC1 $60.22 \%$

Figure 2. Principal component analysis of biomass and growth data of Apuleia leiocarpa seedlings grown in inoculated and uninoculated substrates.

Figura 2. Análise de Componentes Principais das variáveis de biomassa e crescimento mensuradas em experimento com a espécie A. leiocarpa.

Data are the mean of six replicate determinations. D, diameter; H, height; SDW, shoot dry weight; RDW, root dry weight; DQI, Dickson quality index; $\mathrm{M}$, mycorrhizal colonization; $\mathrm{T}_{1}, 60 \%$ Cambisol $+10 \%$ Biomix $+10 \%$ sand $+20 \%$ vermiculite, uninoculated; $\mathrm{T}_{2}, 60 \%$ Cambisol $+10 \%$ Biomix $+10 \%$ sand $+20 \%$ vermiculite + mycorrhizal inoculum; $\mathrm{T}_{3}, 80 \%$ Cambisol $+20 \%$ Biomix, uninoculated; $\mathrm{T}_{4}, 80 \%$ Cambisol + $20 \%$ Biomix + mycorrhizal inoculum; $\mathrm{T}_{5}, 50 \%$ Cambisol $+30 \%$ Biomix $+10 \%$ sand $+10 \%$ vermiculite, uninoculated; $\mathrm{T}_{6}, 50 \%$ Cambisol + $30 \%$ Biomix $+10 \%$ sand $+10 \%$ vermiculite + mycorrhizal inoculum $; \mathrm{T}_{7}, 60 \%$ Cambisol $+40 \%$ Biomix, uninoculated; $\mathrm{T}_{8}, 60 \%$ Cambisol + $40 \%$ Biomix + mycorrhizal inoculum; $\mathrm{T}_{9}, 70 \%$ Cambisol $+30 \%$ bovine manure, uninoculated; $\mathrm{T}_{10}, 70 \%$ Cambisol $+30 \%$ bovine manure + mycorrhizal inoculum; T11, Cambisol fertilized with phosphorus, uninoculated; $\mathrm{T}_{12}$, Cambisol fertilized with phosphorus + mycorrhizal inoculum.

Note in Figures 1 and 2 that plant height and diameter show no correlation with mycorrhizal inoculation for $P$. grandiflorum but a weak association for A. leiocarpa. Such discrepancy stems from ecological factors and differences in nutrient requirements between species.

\section{Nutritional status}

$\mathrm{K}$ content was not influenced by mycorrhizal inoculation; no differences were observed between treatments. $\mathrm{N}$ levels were significantly higher in $\mathrm{T}_{10}$ for both species, as bovine manure contains high levels of this nutrient. Mycorrhizal inoculation increased P levels, regardless of substrate. The highest P concentration was

FLORESTA, Curitiba, PR, v. 49, n. 4, p. 623 - 632, out/dez 2019. 
observed in $\mathrm{T}_{10}$ (Table 6), showing that mycorrhizal inoculation increases $\mathrm{P}$ uptake by plants (OLIVEIRA JÚNIOR et al., 2017).

Table 6. Interaction effects between substrate and mycorrhizal inoculation on the nutritional status of Pseudobombax grandiflorum and Apuleia leiocarpa seedlings.

Tabela 6. Efeito da interação entre os substratos utilizados e os fungos micorrízicos no estado nutricional das espécies $P$. grandiflorum e A. leiocarpa.

\begin{tabular}{|c|c|c|c|c|c|c|c|c|c|c|c|c|}
\hline \multirow{3}{*}{$\begin{array}{c}\text { Treatment } \\
\mathrm{T}_{1}\end{array}$} & \multicolumn{6}{|c|}{ P. grandiflorum } & \multicolumn{6}{|c|}{ A. leiocarpa } \\
\hline & \multicolumn{2}{|c|}{$\mathrm{N}(\%)$} & \multicolumn{2}{|c|}{$\mathrm{P}(\%)$} & \multicolumn{2}{|c|}{$\mathrm{K}(\%)$} & \multicolumn{2}{|c|}{$\mathrm{N}(\%)$} & \multicolumn{2}{|c|}{$\mathrm{P}(\%)$} & \multicolumn{2}{|c|}{$\mathrm{K}(\%)$} \\
\hline & 0.42 & $\mathrm{~b}$ & 0.19 & $\mathrm{~b}$ & 0.69 & $\mathrm{a}$ & 0.32 & $\mathrm{~b}$ & 0.09 & $\mathrm{~b}$ & 0.58 & $\mathrm{a}$ \\
\hline $\mathrm{T}_{2}$ & 0.58 & $\mathrm{~b}$ & 0.23 & $\mathrm{a}$ & 0.91 & $\mathrm{a}$ & 0.47 & $\mathrm{~b}$ & 0.09 & $b$ & 0.80 & $\mathrm{a}$ \\
\hline $\mathrm{T}_{3}$ & 0.33 & $\mathrm{~b}$ & 0.17 & $\mathrm{~b}$ & 0.62 & $\mathrm{a}$ & 0.23 & $\mathrm{~b}$ & 0.06 & $\mathrm{~b}$ & 0.37 & $\mathrm{a}$ \\
\hline $\mathrm{T}_{4}$ & 0.56 & $\mathrm{~b}$ & 0.24 & $\mathrm{a}$ & 0.48 & $\mathrm{a}$ & 0.46 & $\mathrm{~b}$ & 0.13 & $\mathrm{a}$ & 0.51 & $\mathrm{a}$ \\
\hline $\mathrm{T}_{5}$ & 0.39 & $\mathrm{~b}$ & 0.15 & $b$ & 0.64 & $\mathrm{a}$ & 0.29 & $\mathrm{~b}$ & 0.07 & $\mathrm{~b}$ & 0.53 & $\mathrm{a}$ \\
\hline $\mathrm{T}_{6}$ & 0.51 & $\mathrm{~b}$ & 0.25 & $\mathrm{a}$ & 0.55 & $\mathrm{a}$ & 0.41 & $\mathrm{~b}$ & 0.13 & $\mathrm{a}$ & 0.44 & $\mathrm{a}$ \\
\hline $\mathrm{T}_{7}$ & 0.35 & $\mathrm{~b}$ & 0.18 & $b$ & 0.54 & $\mathrm{a}$ & 0.39 & $\mathrm{~b}$ & 0.07 & $\mathrm{~b}$ & 0.44 & $\mathrm{a}$ \\
\hline $\mathrm{T}_{8}$ & 0.50 & $\mathrm{~b}$ & 0.26 & $\mathrm{a}$ & 0.50 & $\mathrm{a}$ & 0.24 & $\mathrm{~b}$ & 0.13 & $\mathrm{a}$ & 0.40 & $\mathrm{a}$ \\
\hline $\mathrm{T}_{9}$ & 0.46 & $\mathrm{~b}$ & 0.14 & $b$ & 0.56 & $\mathrm{a}$ & 0.35 & $b$ & 0.04 & $\mathrm{~b}$ & 0.45 & $\mathrm{a}$ \\
\hline $\mathrm{T}_{10}$ & 0.91 & $\mathrm{a}$ & 0.31 & $\mathrm{a}$ & 0.86 & $\mathrm{a}$ & 0.69 & a & 0.14 & $\mathrm{a}$ & 0.75 & a \\
\hline $\mathrm{T}_{11}$ & 0.33 & $\mathrm{~b}$ & 0.14 & $\mathrm{~b}$ & 0.58 & $\mathrm{a}$ & 0.19 & $\mathrm{~b}$ & 0.04 & $\mathrm{~b}$ & 0.48 & $\mathrm{a}$ \\
\hline $\mathrm{T}_{12}$ & 0.38 & $\mathrm{~b}$ & 0.18 & $\mathrm{~b}$ & 0.59 & $\mathrm{a}$ & 0.27 & $\mathrm{~b}$ & 0.12 & $\mathrm{a}$ & 0.48 & $\mathrm{a}$ \\
\hline $\mathrm{CV}(\%)$ & 32.55 & & 25.1 & & 51.02 & & 38.57 & & 39.22 & & 61.68 & \\
\hline
\end{tabular}

Means in a column followed by different letters differ significantly by the Scott-Knott test $(p<0.05) . \mathrm{T}_{1}, 60 \% \mathrm{Cambisol}+10 \% \mathrm{Biomix}+10 \%$ sand $+20 \%$ vermiculite, uninoculated; $\mathrm{T}_{2}, 60 \%$ Cambisol $+10 \%$ Biomix $+10 \%$ sand $+20 \%$ vermiculite + mycorrhizal inoculum; $\mathrm{T}_{3}, 80 \%$ Cambisol $+20 \%$ Biomix, uninoculated; $\mathrm{T}_{4}, 80 \%$ Cambisol $+20 \%$ Biomix + mycorrhizal inoculum; $\mathrm{T}_{5}, 50 \% \mathrm{Cambisol}+30 \% \mathrm{Biomix}+10 \%$ sand $+10 \%$ vermiculite, uninoculated. $\mathrm{T}_{6}, 50 \%$ Cambisol $+30 \%$ Biomix $+10 \%$ sand $+10 \%$ vermiculite + mycorrhizal inoculum; $\mathrm{T}_{7}, 60 \%$ Cambisol $+40 \%$ Biomix, uninoculated. $\mathrm{T}_{8}, 60 \%$ Cambisol $+40 \%$ Biomix + mycorrhizal inoculum; $\mathrm{T}_{9}, 70 \%$ Cambisol $+30 \%$ bovine manure, uninoculated; $\mathrm{T}_{10}, 70 \%$ Cambisol $+30 \%$ bovine manure + mycorrhiza; $\mathrm{T} 11,100 \%$ Cambisol fertilized with phosphorus, uninoculated. $\mathrm{T}_{12}$, $100 \%$ Cambisol fertilized with phosphorus + mycorrhizal inoculum; CV, coefficient of variation; N, nitrogen; P, phosphorus; K, potassium.

\section{DISCUSSION}

\section{Seedling response to inoculation}

The main and interaction effects of substrate and mycorrhizal inoculation on growth parameters were significant for both species, showing their importance for seedling development. The difference in colonization percentage observed in A. leiocarpa corroborates the findings of Oliveira Júnior et al. (2017), who showed that the species is dependent of mycorrhiza and is capable of forming a strong symbiosis that is highly resistant to changes in environmental conditions. For, A. leiocarpa the results suggest that the high levels of $\mathrm{N}$ in bovine manure favored plant growth.

Overall, the best results were obtained using Cambisol, bovine manure, and mycorrhizal fungi $\left(\mathrm{T}_{10}\right)$ and $80 \%$ Cambisol, 20\% Biomix, and mycorrhizal fungi $\left(\mathrm{T}_{4}\right)$. These results were likely due to the physical and chemical characteristics ( $\mathrm{pH}, \mathrm{N}$ and $\mathrm{P}$ levels) of the substrates, which contributed to seedling growth and mycorrhizal symbiosis. In general, inoculated plants had higher heights, but no relationship was found between plant height and mycorrhizal colonization.

\section{Seedling quality}

Treatments $\mathrm{T}_{3}, \mathrm{~T}_{4}, \mathrm{~T}_{10}$ and $\mathrm{T}_{11}$ promoted plant growth and resulted in improved seedling quality, according to criteria proposed by Caldeira et al. (2008). The authors also recommended maintaining an H/D ratio below 10 during seedling development; all $P$. grandiflorum and A. leiocarpa seedlings were within the recommended range of H/D ratio. This parameter is known to be influenced by inherent species traits, seedling age, substrate composition, pot volume, and nursery practices.

The highest H/SDW index for $P$. grandiflorum was observed in $\mathrm{T}_{9}(70 \%$ Cambisol $+30 \%$ bovine manure, uninoculated), indicating reduced biomass gain, lignification, and quality (Table 4).

A. leiocarpa seedlings grown in uninoculated Cambisol fertilized with $\mathrm{P}\left(\mathrm{T}_{11}\right)$ had a very high $\mathrm{H} / \mathrm{SDW}$ index and showed signs of etiolation. Mycorrhizal inoculation $\left(\mathrm{T}_{12}\right)$ decreased the H/SDW index by a factor of 4 , indicating that mycorrhizal fungi contributed to nutrient uptake and healthy growth (Table 5). Gomes et al. (2013) 
considered H/SDW values below 2 to be indicative of greater survival capacity, regardless of tree species, because the H/SDW index is inversely proportional to lignification. High SDW/RDW ratios indicate a growth imbalance that may result in low resistance to environmental conditions, deficient water absorption, and decreased survival (GOMES et al., 2013). Based on important morphological characteristics for seedling quality, the DQI provides a balanced measure of plant development, robustness, and biomass distribution. The index is affected by species, substrate composition, production and management practices, type of container, and plant age (CALDEIRA et al., 2008). The results are evidence of the beneficial effects of mycorrhizal fungi on the development and quality of the studied plants. Tavares et al. (2016) reported positive results with mycorrhizal inoculation of Acacia mangium Willd for seedling production in non-sterilized Cerrado soil, concluding that beneficial fungi improved seedling growth, nutrition, and quality

\section{Nutritional status}

It is well established that mycorrhizal fungi enhance mineral nutrition in plants. Dias et al. (2012) evaluated the effects of inoculation with mycorrhizal fungi and rhizobia on the growth and nutrition of Anadenanthera macrocarpa progeny. The authors observed that co-inoculation increased absorption of $\mathrm{N}$ and $\mathrm{P}$ by seedlings compared with uninoculated plants, producing quality seedlings at a lower cost.

In the present study, the combination of Cambisol, bovine manure, and mycorrhizal fungi $\left(\mathrm{T}_{10}\right)$ created a favorable condition for nutrient absorption, particularly for $\mathrm{N}$. Bovine manure can contribute to the physical characteristics of the substrate by increasing aggregate stability and porosity as well as enhancing nutrient supply and moisture retention (OGBONNA et al., 2012).

\section{CONCLUSIONS}

P. grandiflorum and A. leiocarpa seedlings responded differently to different substrates. P. grandiflorum seedlings showed improved growth and quality in $\mathrm{T}_{4}(80 \%$ Cambisol $+20 \%$ Biomix + mycorrhizal inoculum $)$ and $\mathrm{T}_{12}$ (Cambisol fertilized with $\mathrm{P}+$ mycorrhizal inoculum).

For A. leiocarpa, the best treatments were $\mathrm{T}_{4}(80 \%$ Cambisol $+20 \%$ Biomix + mycorrhizal inoculum $), \mathrm{T}_{10}$ $(70 \%$ Cambisol $+30 \%$ bovine manure + mycorrhizal inoculum $)$, and $\mathrm{T}_{12}$ (Cambisol fertilized with $\mathrm{P}+$ mycorrhizal inoculum). Mycorrhizal inoculation was beneficial for seedling growth and quality.

\section{REFERENCES}

CAMARA, R; FONSECA JÚNIOR, A.M; SOUSA, A. C. O., PEREIRA, M. G.; OLIVEIRA JÚNIOR, J.Q. Influência do substrato e inoculação micorrízica na produção de mudas de Colubrina glandulosa Perkins. Floresta, Curitiba, PR, v. 47, n. 4, p.449 - 458, 2017.

CALDEIRA, M. V. W.; ROSA, G. N.; FENILLI, T. A. B.; HARBS, R. M. P. Composto orgânico na produção de mudas de aroeira-vermelha. Scientia Agraria, Curitiba, v. 9, p. 27-33, 2008.

CHAER, G. M.; RESENDE, A. S.; CAMPELlO, E. F. C.; FARIA, S. M.; BODDEY, R. M. Nitrogen-fixing legume tree species for the reclamation of severely degraded lands in Brazil. Tree Physiology, Oxford, v. 31, p.139-149, 2011.

DIAS, P.C.; PEREIRA, M.S.F.; KASUYA, M.C.M.; PAIVA, H.N.; OLIVEIRA, L.S.; XAVIER, A. Micorriza arbuscular e rizóbios no enraizamento e nutrição de mudas de angico-vermelho. Revista Árvore, v. 36, n. 6, p. 1027-1037, 2012.

DICKSON, A.; LEAF, A.L.; HOSNER, J.F. Quality appraisal of white spruce and white pine seedling stock in nurseries. Forest Chronicle, v. 36, n.1 p.10-13, 1960.

FERREIRA, D. F. Sisvar: a computer statistical analysis system. Ciência e Agrotecnologia, Lavras, v. 35, n. 6, p. 1039-1042, nov./dez. 2011.

GIOVANNETTI, M.; MOSSE, B. An evaluation of techniques for measuring vesicular-arbuscular mycorrhizal infection in roots. New Phytologist, Cambridge, v.84, n.3, p. 489-500, 1980.

GOETTEN, L. C.; MORETTO, G.; STURMER, S. L. Influence of arbuscular mycorrhizal fungi inoculum produced on-farm and phosphorus on growth and nutrition of native woody plant species from Brazil. Acta Botanica Brasilica, Belo Horizonte, v. 30, n. 1, p. 9-16, 2016.

GOMES, D. R.; CALDEIRA, M. V. W.; DELARMELINA, W. M.; GONÇALVES, E. O.; TRAZZI, P. A. Lodo de esgoto como substrato para produção de mudas de Tectona grandis L. Cerne, v.19, n.1, p.123-131, 2013.

FLORESTA, Curitiba, PR, v. 49, n. 4, p. 623 - 632, out/dez 2019.

Oliveira Junior, J. Q. et.al.

ISSN eletrônico 1982-4688

DOI: $10.5380 /$ rf.v49 i4.54525 
KOSKE, R. E.; GEMMA, J. N. A modified procedure for staining roots to detect VA mycorrhizas. Mycology Research, Cambridge, v.92 p. 488-505, 1989.

OGBONNA, D. N.; ISIRIMAH, N. O.; PRINCEWILL, E. Effect of organic waste compost and microbial activity on the growth of maize in the Ultisols in Port Harcourt, Nigeria. African Journal of Biotechnology, v. 11, n. 62, p. 12546-12554, 2012.

OLIVEIRA JR., J. Q.; SOUZA, R. C.; FONSECA JUNIOR, A. M.; SOUZA, A. C.; JESUS, E.; PEREIRA, M. G. Dependência e resposta à inoculação com diferentes espécies de fungos micorrízicos arbusculares na espécie florestal Apuleia leiocarpa. Revista Brasileira de Ciência do Solo, Viçosa, v.41, 2017.

SCORIZA, R. N.; CORREIA, M. E. F.; SILVA, E. M. R. Colembolos e fungos micorrizicos arbusculares como indicadores de degradação em fragmentos florestais de encosta. Revista de Ciências Agrárias. Amazonian Journal, v. 59, n. 4, p. 386-392, 2016.

TAVARES, S.R.L.; FRANCO, A.A.; SILVA, E.M.R. Produção de mudas de Acacia mangium Willd noduladas e micorrizadas em diferentes substratos. HOLOS, v. 4, n. 32, 2016.

TEDESCO, M. J; GIANELLO, C; BISSANI, CA. Análise de solo, plantas e outros materiais. 2. Ed. Porto Alegre: Departamento de Solos, UFRGS, p. 177-188. 1995.

THIRKELL, T. J.; CAMERON, D. D.; HODGE, A. Resolving the 'nitrogen paradox' of arbuscular mycorrhizas: fertilization with organic matter brings considerable benefits for plant nutrition and growth. Plant, Cell \& Environment, v. 39, p. 1683-1690, 2016. 